\title{
Evaluating the Influence of Topography on Species Diversity, Distribution and Composition of Forests in Central Vietnam
}

\author{
Hung Nguyen Trong ${ }^{1,2 *}$, Tung Pham Gia ${ }^{1,3}$ and Martin Kappas ${ }^{1}$ \\ 'Cartography, GIS and Remote Sensing Department, Göttingen University, Germany; mkappas@gwdg.de \\ ${ }^{2}$ Ministry of Natural Resources and Environment, Hanoi, Vietnam; tronghung2128@gmail.com \\ 3 University of Agriculture and Forestry, Hue University, Vietnam; phamgiatung@huaf.edu.vn
}

\begin{abstract}
Objectives: This study focused on evaluating the influence of topography conditions on species richness, diversity, distribution and species composition as dependent variables at different micro topographic attributes in Central Vietnam. Methods/Statistical Analysis: Trees with diameter at breast height $\geq 6.0 \mathrm{~cm}$ of 90 quadratic plots $\left(1000 \mathrm{~m}^{2}\right)$ were inventoried to assess species richness, diversity, and distribution. Scatterplot Matrices: Pairs-sample Test was applied to evaluate the multiple correlations between ranked elevations and slopes with dependent variables. The composition of most abundant, dominant species including Importance Value Index at the midpoint of each topographic attribute was compared with those of the entire topographic attribute. Findings: A total of 4297 tree individuals were recorded representing 122 species from 47 families. We found a significant difference of species richness, diversity, stem density and basal area in different topographic attributes $(\mathrm{p}<0.05)$. The lower elevation and shallower slope had more species richness and diversity than those in the higher elevation and steeper slope. Elevation had more influence on the distribution of species, stand density, species diversity, basal area and family than those of the slope. The species composition between slopes was not remarkably distinguishable which helps to confirm that slopes do not contribute to species distribution, composition. Fagaceae, Myrtaceae, Lauraceae, Cannabaceae, Meliaceae, Sapindaceae, Dipterocarpaceae, Leguminosae, Burseraceae and Malvaceae were the most dominant. The composition of the most abundant and dominant species at the midpoint plots objected to those of the entire topographic attribute. Difference of species composition between elevations may have subjected due to disturbance or successional processes of different forest ecological habitats in the current study area. Application/Improvements: Mapping micro-site natural forest disturbances based topographic conditions of dominant, endemic species for conservation and management of different ecological habitats with support of highresolution satellite images.
\end{abstract}

Keywords: Composition, Central Vietnam, Species Diversity, Topography

\section{Introduction}

Tropical forests host the most species richness and complex plant communities ofall forests ${ }^{1,2}$. Species diversity is an indicator that shows substantial links between the richness and abundance of individual tree species, reflecting the heterogeneity or existence of vegetation ${ }^{3}$.

The diversity of tree species is fundamental to entire forest biodiversity because individuals of tree species

${ }^{*}$ Author for correspondence give resources and formation of habitat for the forest ecosystem ${ }^{4-6}$. Factors that influence forest species richness at different scales could be comprising soil, parent materials, air temperature, light and even altitudes ${ }^{?}$. The spatial distribution of trees in the tropical forest ecosystem or forest habitat has been a major interest for plant ecologists because of its potential role in explaining the coexistence of different tree species in species-rich forests ${ }^{-}$. Understanding forest species richness, diversity, 
species distribution in the current study area where the taxon spatially aligned and species composition in where different species are favored in a community, is necessary for forest sustainability, species conservation, management of forest ecosystems, and natural forest landscape restoration $\%$.

Tree species composition is also a very important indicator for assessing the Importance Value Index (IVI) of a species within a forest stand, providing information on abundance, dominance and frequency thereby providing information on the contribution of the species to vegetation formation ${ }^{10}$. Natural forests in Vietnam have declined dramatically as a result of the Vietnam War, population growth, overexploitation, and the transformation of forested areas into arable land $\mathrm{d}^{11}$. Forest covers of Vietnam was around 43\% in 1943 and declined to below $28 \%$ in $1990 \mathrm{~s}^{\frac{12}{2}}$. The forests of the Northern and Southern Truong Son Mountain Range have been highlighted as global eco-regions by the World Wide Fund for Nature ${ }^{13}$. The tropical evergreen forests are geographically classified below $700 \mathrm{~m}$ a.s.l. (above sea level) in the north and below $1000 \mathrm{~m}$ a.s.l. in the south. The subtropical evergreen forests are classified above $700 \mathrm{~m}$ a.s.l. in the north and above $1000 \mathrm{~m}$ a.s.l. in the south of Vietnam. Vegetation communities are further identified by edaphic condition, the level of disturbance and floristics, and finally by the dominant species.

Numerous researchers have documented that plant species composition and abundance are related to the heterogeneity of soil properties, topography, and stage (i.e. age) of forest succession $\frac{14-19}{}$. In Vietnam, some studies have simply described the spatial distribution of trees in forests ${ }^{20,21}$. While the influence of topographic conditions as topographic attributes on tree diversity, composition and distribution has not been studied 22 , no study has been conducted on the influence of topographic features (such as elevations and slopes) on tree species distribution, species diversity and composition.

To fill the gapped knowledge about the tropical evergreen forests in the Northern Central Truong Son Mountain Range in the Central Vietnam, this study aims to evaluate the influence of topographic attributes on species richness, species distribution and to compare the species composition of the most dominant species of the representative plots with those of the entire topographic attribute respectively.
The study area is located in the North Central Truong Son Mountain Range and of the A Luoi district in Vietnam (Figure 1), which borders ${ }^{23}$ from $107^{\circ} \mathrm{E}$ to $107^{\circ} 30^{\prime} \mathrm{E}$ and from $16^{\circ} \mathrm{N}$ to $16^{\circ} 30^{\prime} \mathrm{N}$ in the western part of the Thua Thien Hue province. The climate consists typical tropical monsoon characteristics. The area is frequently influenced by an intertropical convergence zone that typically causes tropical low pressures and typhoons, leading to annual rainfall of about $3500 \mathrm{~mm}$ with an average of 200 rainy days per year, most of the rainy days accumulate between September and December ${ }^{21}$. The average annual temperature is $25^{\circ} \mathrm{C}$, the average high temperature is $26.1^{\circ} \mathrm{C}$, and the average low temperature is $18.5^{\circ} \mathrm{C}$. Moisture content varies from $86 \%$ to $96 \%$. The typical topography in this mountainous region features complex, steep slopes ranging from 5 to $48^{\circ}$. The elevation of the study area is from 150 to $1162 \mathrm{~m}$ a.s.l thus the landscape of A Luoi is usually affected by landslides $\stackrel{24}{ }$.

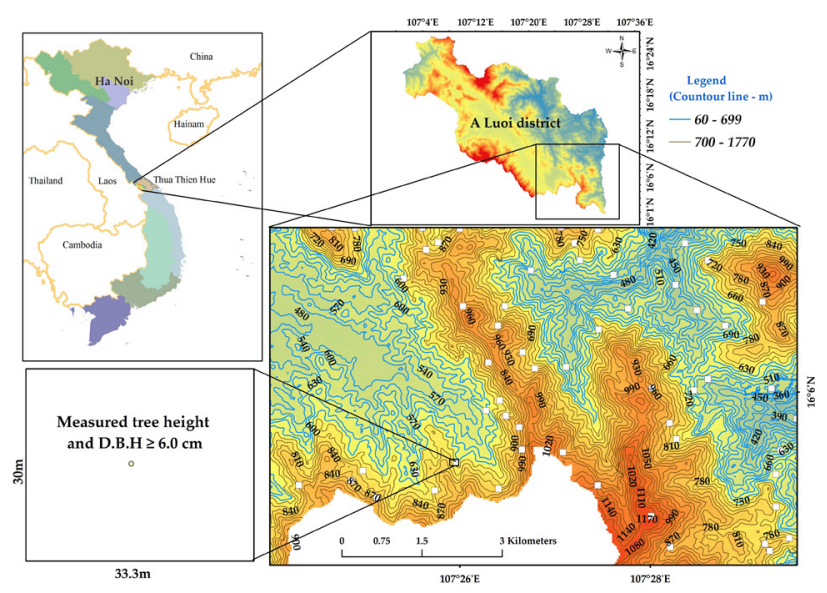

Figure 1. Study area and sample plot design.

The primary and secondary lowland evergreen forests are consisted in the current study area ${ }^{25}$. The most dominant genera of Dipterocarpus, Hopea, Parashorea of Dipterocarpceae and Lithocarpus and Castanopsis of Fagaceae and followed by Syzygium of Myrtaceae; as well as the genera of Lauraceae, namely Machilus, Litsea and Cinamomum, and of the Cannabaceae was Gironniera, Aphananthe. Forests in the study area are under management of Sao La Nature Reserve and Management Board of Watershed Forests. Four soil types are present within the research area: Ferralic Acrisols (75\%), Arenic Acrisols (14\%), Humic Acrisols (6\%), and Hyperdystrict Acrisols (5\%) $\frac{26}{6}$. 


\section{Materials and Methods}

\subsection{Vegetation sampling and Topographic attribute classification}

A total of 90 plots $(30 \times 33.3 \mathrm{~m})$ were randomly sampled in the natural forests of the study area (Figure 1). The top height $(\mathrm{Ht})$ in meters and the Diameter at Breast Height (DBH) of all living trees with a $\mathrm{DBH} \geq 6.0 \mathrm{~cm}$ were recorded for the calculation of the mean stand parameters ${ }^{27,28}$. The tree species were recorded in the field in the Vietnamese language and later translated to their scientific names. The names of all tree species were checked to avoid synonyms in the Plantlist $\frac{29}{}$. Coordinates, elevations, and slopes of all sample plots were recorded on-site with GPS and calibrated with a Global Digital Elevation Model (DEM) resolution $(30 \times 30 \mathrm{~m})$ in ArcGIS 10.5. The minimum interval distance between plots was approximately $200 \mathrm{~m}$.

As forests in Vietnam were ecologically classified, we divided the topographic elevation into two levels, $\mathrm{H} 1$ (150-696 m a.s.l.) and H2 (700-1162 m a.s.l.). For slope classification, we took into account of studies of $\frac{24,30,31}{}$ and we decide to divide slopes into two levels, lower slope (S1) ranged from 5 to 20 degrees, and steeper slope (S2) ranged from 20 to 48 degrees.

\subsection{Species richness and species diversity distribution}

In general, higher values of biodiversity indices such as species diversity and species dominance indicate large species richness ${ }^{32}$. The Shannon Index $(\mathrm{H})$ of species was calculated as ${ }^{33}$ :

$$
\text { Shannon } \operatorname{Index}(H)=-\sum_{i=1}^{s} p_{i} \ln \left(p_{i}\right)
$$

where, $p^{i}$ is the proportion of $S$ made up of the $i^{\text {th }}$ species, and the Evenness (Eq) was calculated based ${ }^{34}$ as:

$$
\mathrm{Eq}=\frac{H}{\ln (S)}
$$

where, $\mathrm{H}$ is Shannon Index and $\ln (S)$ is the natural logarithm of the total number of species in the attribute.

We calculated the mean number of species, number of families, stem density, and basal area of all sampled plots for each topographic attribute to assess the species richness, stem density, dominance, correlation between elevations, and slope and species distribution under different topography.

Statistical 13.3 was used to test for significant differences between the mean numbers of species, number of families and stem density; and the basal area, dominance and abundance of species. In order to present multiple regressions between topographic attributes and forest stand variables, the Scatter Plot Matrices (SPLOMS) with pairs. panels package in R (RStudio Team, 2015) was used $\frac{35}{}$. We then compared the means of species, individuals among topographic attributes and stand parameters ${ }^{36}$. The forest parameters of the current study are presented in Table 1.

\subsection{Species Composition}

Along with assessing the species composition for all topographic attributes, we also compared the contributions of dominance and abundance of the most ten species that

\begin{tabular}{|c|c|c|c|c|}
\hline \multirow[b]{2}{*}{ Attributes/Parameters } & \multicolumn{2}{|c|}{ Elevations (m) a.s.l. } & \multicolumn{2}{|c|}{ Slopes (degree) } \\
\hline & $\begin{array}{c}\text { H1 } \\
(150-699) \\
\end{array}$ & $\begin{array}{c}\mathrm{H} 2 \\
(700-1162)\end{array}$ & $\begin{array}{c}\text { S1 } \\
(5-20) \\
\end{array}$ & $\begin{array}{c}\mathrm{S} 2 \\
(20-48) \\
\end{array}$ \\
\hline No. of Plots $\left(1000 \mathrm{~m}^{2}\right)$ & 50 & 40 & 38 & 52 \\
\hline Mean topographic attribute $( \pm \mathrm{SD} /$ attr $)$ & $527 \pm 146 a$ & $808 \pm 92 b$ & $14.3 \pm 4.7 \mathrm{a}$ & $28.1 \pm 5.8 b$ \\
\hline Species richness $( \pm \mathrm{SD})$ (species/plot) & $29.7 \pm 3.4 \mathrm{a}$ & $20.2 \pm 3.6 b$ & $27.1 \pm 5.5 \mathrm{a}$ & $23.8 \pm 5.8 b$ \\
\hline Families $( \pm \mathrm{SD})$ (family/plot) & $21.3 \pm 2.9 \mathrm{a}$ & $15.8 \pm 2.8 \mathrm{~b}$ & $19.6 \pm 3.9 \mathrm{a}$ & $18.0 \pm 3.8 \mathrm{a}$ \\
\hline Stand density $( \pm \mathrm{SD})($ stem $/ \mathrm{ha})$ & $527 \pm 8.9 \mathrm{a}$ & $415 \pm 13.2 b$ & $508 \pm 10.6 \mathrm{a}$ & $455 \pm 13.7 \mathrm{~b}$ \\
\hline Basal Area $( \pm \mathrm{SD})\left(\mathrm{m}^{2} / \mathrm{ha}\right)$ & $21.8 \pm 0.6 \mathrm{~b}$ & $26.2 \pm 1.0 \mathrm{a}$ & $21.5 \pm 0.8 \mathrm{a}$ & $25.4 \pm 0.8 \mathrm{~b}$ \\
\hline Shannon Index $(\mathrm{H})$ & $3.2 \pm 0.14 \mathrm{a}$ & $2.7 \pm 0.20 \mathrm{~b}$ & $3.1 \pm 0.29 \mathrm{a}$ & $2.9 \pm 0.26 \mathrm{~b}$ \\
\hline Equality (Eq) & $94 \pm 0.02 \mathrm{a}$ & $93 \pm 0.03 b$ & $98 \pm 0.31 \mathrm{a}$ & $93 \pm 0.05 \mathrm{a}$ \\
\hline
\end{tabular}

Table 1. Species richness, diversity, density over respective topographic attributes

(Within column, the values followed by the same letter $(\mathrm{a}, \mathrm{b})$ are not significantly different $(\mathrm{p}<0.05)$ between $\mathrm{H} 1$ and $\mathrm{H} 2$ of each parameter and values followed by the same letter $(\mathrm{a}, \mathrm{b})$ are not significantly different $(\mathrm{p}<0.05)$ between S1 and S2 of each parameter) 
were present in the representative plots to those of the entire attribute. The species composition of tree species as of species abundance as number of individuals (N/ha), absolute dominance as basal area $\left(\mathrm{m}^{2} / \mathrm{ha}\right)$, frequency in percent (\%), and IVI of each species where consists of relative density, relative frequency and relative dominance of the attribute were calculated 20,37 . The comparison of species composition aimed at accessing the contribution of the ten most dominant species which appeared in the representative plots of each topographic attribute with those of the entire attribute with the hope to differentiate different forest ecology habitats where different species were compositionally found in different topographic attribute (Table 2).

Table 2. Species composition of the representative plots and of the entire topographic attribute

\begin{tabular}{|c|c|c|c|c|c|c|c|c|}
\hline \multirow[b]{2}{*}{ Species } & \multirow[b]{2}{*}{ Family } & \multicolumn{3}{|c|}{ Representative Plots } & \multicolumn{4}{|c|}{ Topographic Attributes } \\
\hline & & 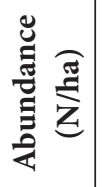 & 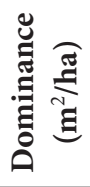 & 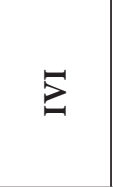 & 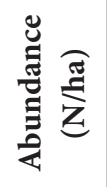 & 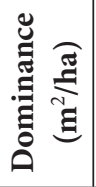 & $\frac{\widehat{e}}{e^{e}}$ & $\sum$ \\
\hline Syzygium zeylanicum (L.) DC. (5:63) & Myrtaceae & 1.00 & 0.06 & 24.62 & 12.60 & 0.71 & 66 & 6.07 \\
\hline Bursera tonkinensis Guillaum (4:57) & Burseraceae & 0.80 & 0.12 & 33.82 & 11.40 & 0.89 & 46 & 6.21 \\
\hline Dipterocarpus grandiflorus Blanco (3:46) & Dipterocarpaceae & 0.60 & 0.02 & 13.06 & 9.20 & 0.33 & 42 & 4.21 \\
\hline Shorea roxburghii G.Don (2:26) & Dipterocarpaceae & 0.40 & 0.04 & 14.35 & 5.20 & 0.37 & 34 & 3.54 \\
\hline Alangium ridleyi King (2:63) & Cornaceae & 0.40 & 0.03 & 13.34 & 12.60 & 0.49 & 60 & 5.54 \\
\hline Wrightia annamensis Eberh. \& Dubard (2:36) & Apocynaceae & 0.40 & 0.02 & 10.46 & 7.20 & 0.18 & 44 & 3.42 \\
\hline Artocarpus rigidus Blume (1:14) & Moraceae & 0.20 & 0.07 & 18.49 & 2.80 & 0.16 & 14 & 2.46 \\
\hline Amesiodeuchon chinense (Merr.) Hu (1:19) & Sapindaceae & 0.20 & 0.05 & 13.70 & 3.80 & 0.31 & 28 & 3.07 \\
\hline Artocarpus tonkinensis A.Chev. ex Gagnep. (1:20) & Moraceae & 0.20 & 0.02 & 9.58 & 4.00 & 0.21 & 24 & 2.67 \\
\hline Engelhardtia roxburghiana Lindl (1:46) & Juglandaceae & 0.20 & 0.02 & 8.14 & 9.20 & 0.63 & 46 & 5.08 \\
\hline H1: Other (34:390) & & 4.40 & 0.12 & 140.44 & 449.00 & 17.53 & - & 231.00 \\
\hline H1: Total (44:2635) & & 8.80 & 0.55 & 300.00 & 527.00 & 21.80 & - & 300.00 \\
\hline Elaeocarpus sylvestris (Lour.) Poir. (7:23) & Elaeocarpaceae & 1.75 & 0.13 & 37.41 & 5.75 & 0.40 & 20 & 3.85 \\
\hline Gironniera subaequalis Planch (4:69) & Cannabaceae & 1.00 & 0.08 & 24.22 & 17.25 & 0.80 & 58 & 8.02 \\
\hline Lithocarpus ducampii (Hickel \& A.Camus) (4:110) & Fagaceae & 1.00 & 0.08 & 23.92 & 27.50 & 2.02 & 60 & 12.06 \\
\hline $\begin{array}{l}\text { Scaphium macropodum (Miq) Beumee ex K.Heyne } \\
(3: 52)\end{array}$ & Malvaceae & 0.75 & 0.05 & 17.51 & 13.00 & 0.51 & 68 & 7.02 \\
\hline Helicia cochinchinensis Lour. (3:30) & Proteaceae & 0.75 & 0.01 & 13.08 & 7.50 & 0.29 & 33 & 4.07 \\
\hline Macaranga denticulata (Blume) (2:18) & Euphorbiaceae & 0.50 & 0.04 & 14.07 & 4.50 & 0.12 & 25 & 2.66 \\
\hline Knema elegans Warb (2:23) & Myristicaceae & 0.50 & 0.02 & 12.05 & 5.75 & 0.30 & 23 & 3.47 \\
\hline Vitex quinata (Lour.) F.N.Williams (2:7) & Lamiaceae & 0.50 & 0.01 & 11.28 & 1.75 & 0.05 & 15 & 1.41 \\
\hline Canarium album (Lour.) DC. (2:26) & Burseraceae & 0.50 & 0.01 & 11.10 & 6.50 & 0.35 & 43 & 4.27 \\
\hline Cinnamomum verum J.Presl (2:5) & Lauraceae & 0.50 & 0.01 & 11.09 & 1.25 & 0.03 & 10 & 0.99 \\
\hline H2: Other (33:363) & & 3.00 & 0.35 & 124.28 & 324.75 & 21.38 & - & 252.16 \\
\hline H2: Total (43:1662) & & 10.75 & 0.79 & 300.00 & 415.50 & 26.25 & - & 300.00 \\
\hline Lithocarpus tubulosus (Hickel \& A.Camus) (11:37) & Fagaceae & 2.89 & 0.02 & 30.11 & 9.74 & 0.51 & 34 & 5.03 \\
\hline Artocarpus tonkinensis A.Chev. ex Gagnep. (3:18) & Moraceae & 0.79 & 0.00 & 18.23 & 4.74 & 0.28 & 24 & 3.26 \\
\hline Aphanamixis grandiflora (3:42) & Euphorbiaceae & 0.79 & 0.01 & 14.69 & 11.05 & 0.41 & 58 & 5.19 \\
\hline Endospermun sinensis Benth. (3:21) & Euphorbiaceae & 0.79 & 0.01 & 13.38 & 5.53 & 0.16 & 29 & 2.82 \\
\hline Symplocos glauca (Thunb.) Koidz. (3:41) & Symplocaceae & 0.79 & 0.01 & 10.66 & 10.79 & 0.39 & 47 & 4.89 \\
\hline
\end{tabular}




\begin{tabular}{|c|c|c|c|c|c|c|c|c|}
\hline \multirow[b]{2}{*}{ Species } & \multirow[b]{2}{*}{ Family } & \multicolumn{3}{|c|}{ Representative Plots } & \multicolumn{4}{|c|}{ Topographic Attributes } \\
\hline & & 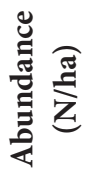 & 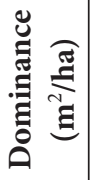 & $\Sigma$ & 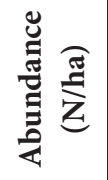 & 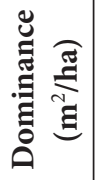 & $\frac{\widehat{\partial}}{\sqrt{e}}$ & 文 \\
\hline Nephelium melliferum Gagnep. (2:39) & Sapindaceae & 0.53 & 0.00 & 9.79 & 10.26 & 0.65 & 58 & 5.54 \\
\hline Prunus arborea (Blume) Kalkman (2:23) & Rosaceae & 0.53 & 0.02 & 9.42 & 6.05 & 0.19 & 34 & 3.15 \\
\hline Syzygium lanceolatum (Lam.) Wight \& Arn (2:73) & Myrtaceae & 0.53 & 0.01 & 8.07 & 19.21 & 0.82 & 79 & 7.98 \\
\hline Adina pilulifra (1:11) & Rubiaceae & 0.26 & 0.01 & 9.72 & 2.89 & 0.06 & 24 & 1.78 \\
\hline Alphonsea monogyna Merr.\&Chun (1:6) & Annonaceae & 0.26 & 0.02 & 7.94 & 1.58 & 0.04 & 11 & 1.18 \\
\hline S1: Other (36:311) & & 3.95 & 0.70 & 167.99 & 426.32 & 17.99 & - & 259.17 \\
\hline S1: Total (46:1931) & & 12.11 & 0.80 & 300.00 & 508.16 & 21.50 & - & 300.00 \\
\hline Lithocarpus ducampii (Hickel \& A.Camus) (5:128) & Fagaceae & 0.96 & 0.09 & 31.99 & 24.62 & 1.86 & 67 & 10.55 \\
\hline Engelhardtia roxburghiana Lindl (3:51) & Juglandaceae & 0.58 & 0.04 & 16.75 & 9.81 & 0.83 & 46 & 5.61 \\
\hline Ormosia pinnata (Lour.) Merr (3:29) & leguminosae & 0.58 & 0.03 & 15.66 & 5.58 & 0.28 & 40 & 3.51 \\
\hline Sapium discolor (3:11) & Euphorbiaceae & 0.58 & 0.03 & 14.39 & 2.12 & 0.10 & 13 & 1.67 \\
\hline Gironniera subaequalis Planch (3:79) & Cannabaceae & 0.58 & 0.01 & 10.98 & 15.19 & 0.62 & 60 & 6.72 \\
\hline Archidendron clypearia (Jack) I.C.Nielsen (3:10) & Leguminosae & 0.58 & 0.01 & 10.29 & 1.92 & 0.07 & 12 & 1.39 \\
\hline Canarium album (Lour.) DC. (3:39) & Burseraceae & 0.58 & 0.01 & 9.83 & 7.50 & 0.27 & 42 & 3.98 \\
\hline Aphananthe cuspidata (Blume) Planch (2:49) & Cannabaceae & 0.38 & 0.04 & 15.55 & 9.42 & 0.52 & 38 & 4.81 \\
\hline Diospyros sylvatica Roxb (2:24) & Ebenaceae & 0.38 & 0.01 & 8.71 & 4.62 & 0.33 & 23 & 3.16 \\
\hline Aphanamixis grandiflora (2:64) & Meliaceae & 0.38 & 0.00 & 7.14 & 12.31 & 0.44 & 60 & 5.84 \\
\hline S2: Other (50:484) & & 5.96 & 0.15 & 158.73 & 361.92 & 20.12 & - & 252.76 \\
\hline S2: Total (60:2366) & & 11.54 & 0.42 & 300.00 & 455.00 & 25.44 & - & 300.00 \\
\hline
\end{tabular}

(Species presented in bracket i.e: (60:2366) indicating number of individual stems in the representative plot and the individual stems in the topographic attribute; $\mathrm{N}=$ number of stem of a species per ha; F (\%) = Frequency; IVI = Importance Value Index of the representing plot).

\section{Results}

\subsection{Vegetation Distribution over}

\section{Topography}

A total of 4297 individual trees were recorded, representing 122 species from 47 families from the total area $(9.0 \mathrm{ha})$. The ten most abundant species at lower elevation (H1), out of 121 species present, were: Syzygium lanceolatum (Lam.) Wight \& Arn, Lithocarpus ducampii (Hickel \& A.Camus), Lithocarpus dealbatus (Hook.f. \& Thomson ex Miq.) Rehder, Gironniera subaequalis Planch, Syzygium zeylanicum (L.) DC, Alangium ridleyi King, Scaphium macropodum (Miq) Beumee ex K.Heyne, Bursera tonkinensis Guillaum, Lithocarpus tubulosus (Hickel \& A.Camus) and Symplocos glauca (Thunb.) Koidz.
Of these, the three most dominant species were $L$. dealbatus, L. ducampii and B. tonkinensis Guillaum (Table 3).

The ten most dominant species at higher elevation (H2), out of 109 species present, were: Lithocarpus ducampii (Hickel \& A.Camus), Gironniera subaequalis Planch, Lithocarpus tubulosus (Hickel \& A.Camus), Syzygium zeylanicum (L.) DC, Lithocarpus dealbatus (Hook.f. \& Thomson ex Miq.) Rehder, Syzygium zeylanicum (L.) DC, Aphanamixis grandiflora, Scaphium macropodum (Miq) Beumee ex K.Heyne, Alangium ridleyi King and Engelhardtia roxburghiana Lindl. But the most dominant species in $\mathrm{H} 2$ were $L$. ducampii, Shorea roxburghii G.Don, Dipterocarpus grandiflorus Blanco, Bursera tonkinensis Guillaum (Table 3).

The ten most abundant species at shallower slope (S1), out of 116 species present, were: Syzygium lanceolatum 
Table 3. The abundant and dominant species in different topographic attributes

\begin{tabular}{|c|c|c|c|c|c|}
\hline Att & Species & Family & $\mathbf{N}$ & $\begin{array}{c}\text { Abu } \\
\text { (N/ha) }\end{array}$ & $\begin{array}{c}\text { Dom } \\
\left(\mathrm{m}^{2} / \mathbf{h a}\right)\end{array}$ \\
\hline \multirow{12}{*}{$\mathrm{H} 1$} & Syzygium lanceolatum (Lam.) Wight \& Arn & Myrtaceae & 102 & 20.4 & 0.8 \\
\hline & Lithocarpus ducampii (Hickel \& A.Camus) & Fagaceae & 73 & 14.6 & 0.9 \\
\hline & Lithocarpus dealbatus (Hook.f. \& Thomson ex Miq.) Rehder & Fagaceae & 65 & 13.0 & 1.0 \\
\hline & Gironniera subaequalis Planch & Cannabaceae & 65 & 13.0 & 0.4 \\
\hline & Syzygium zeylanicum (L.) DC. & Myrtaceae & 63 & 12.6 & 0.7 \\
\hline & Alangium ridleyi King & Cornaceae & 63 & 12.6 & 0.5 \\
\hline & Scaphium macropodum (Miq) Beumee ex K.Heyne & Malvaceae & 58 & 11.6 & 0.4 \\
\hline & Bursera tonkinensis Guillaum & Burseraceae & 57 & 11.4 & 0.9 \\
\hline & Lithocarpus tubulosus (Hickel \& A.Camus) & Fagaceae & 55 & 11.0 & 0.5 \\
\hline & Symplocos glauca (Thunb.) Koidz. & Symplocaeae & 55 & 11.0 & 0.5 \\
\hline & Total (10) & & 656 & 131.2 & 6.7 \\
\hline & Other (111) & & 1979 & 395.8 & 15.1 \\
\hline \multirow{14}{*}{$\mathrm{H} 2$} & Lithocarpus ducampii (Hickel \& A.Camus) & Fagaceae & 110 & 27.5 & 2.0 \\
\hline & Shorea roxburghii G.Don & Dipterocarpaceae & 69 & 17.3 & 0.8 \\
\hline & Dipterocarpus grandiflorus Blanco & Dipterocarpaceae & 61 & 15.3 & 0.8 \\
\hline & Bursera tonkinensis Guillaum & Burseraceae & 60 & 15.0 & 0.6 \\
\hline & Engelhardtia roxburghiana Lindl & Juglandaceae & 58 & 14.5 & 0.6 \\
\hline & Lithocarpus tubulosus (Hickel \& A.Camus) & Fagaceae & 58 & 14.5 & 0.6 \\
\hline & Gironniera subaequalis Planch & Cannabaceae & 54 & 13.5 & 0.6 \\
\hline & Syzygium zeylanicum (L.) DC. & Myrtaceae & 37 & 9.3 & 0.8 \\
\hline & Lithocarpus dealbatus (Hook.f. \& Thomson ex Miq.) Rehder & Fagaceae & 35 & 8.8 & 0.5 \\
\hline & Aphanamixis grandiflora & Meliaceae & 35 & 8.8 & 0.5 \\
\hline & Alangium ridleyi King & Cornaceae & 21 & 5.3 & 0.6 \\
\hline & Scaphium macropodum (Miq) Beumee ex K.Heyne & Malvaceae & 12 & 3.0 & 0.6 \\
\hline & Total (12) & & 610 & 152.5 & 9.2 \\
\hline & Other (97) & & 1052 & 263.0 & 17.0 \\
\hline \multirow{13}{*}{ S1 } & Lithocarpus dealbatus (Hook.f. \& Thomson ex Miq.) Rehder & Fagaceae & 72 & 18.9 & 1.1 \\
\hline & Syzygium lanceolatum (Lam.) Wight \& Arn & Myrtaceae & 73 & 19.2 & 0.8 \\
\hline & Shorea roxburghii G.Don & Dipterocarpaceae & 21 & 5.5 & 0.8 \\
\hline & Lithocarpus ducampii (Hickel \& A.Camus) & Fagaceae & 55 & 14.5 & 0.8 \\
\hline & Syzygium zeylanicum (L.) DC. & Myrtaceae & 49 & 12.9 & 0.8 \\
\hline & Gironniera subaequalis Planch & Cannabaceae & 55 & 14.5 & 0.6 \\
\hline & Alangium ridleyi King & Cornaceae & 55 & 14.5 & 0.6 \\
\hline & Engelhardtia roxburghiana Lindl & Juglandaceae & 32 & 8.4 & 0.6 \\
\hline & Scaphium macropodum (Miq) Beumee ex K.Heyne & Malvaceae & 48 & 12.6 & 0.5 \\
\hline & Aphanamixis grandiflora & Meliaceae & 42 & 11.1 & 0.4 \\
\hline & Symplocos glauca (Thunb.) Koidz. & Symplocaeae & 41 & 10.8 & 0.4 \\
\hline & Total (11) & & 543 & 142.9 & 7.3 \\
\hline & Other (106) & & 1388 & 365.3 & 14.2 \\
\hline
\end{tabular}




\begin{tabular}{|c|c|c|c|c|c|}
\hline Att & Species & Family & $\mathbf{N}$ & $\begin{array}{c}\text { Abu } \\
\text { (N/ha) }\end{array}$ & $\begin{array}{c}\text { Dom } \\
\left(\mathrm{m}^{2} / \mathbf{h a}\right)\end{array}$ \\
\hline \multirow{15}{*}{ S2 } & Lithocarpus ducampii (Hickel \& A.Camus) & Fagaceae & 128 & 24.6 & 1.9 \\
\hline & Bursera tonkinensis Guillaum & Burseraceae & 28 & 5.4 & 1.1 \\
\hline & Dipterocarpus grandiflorus Blanco & Dipterocarpaceae & 41 & 7.9 & 0.9 \\
\hline & Shorea roxburghii G.Don & Dipterocarpaceae & 30 & 5.8 & 0.9 \\
\hline & Engelhardtia roxburghiana Lindl & Juglandaceae & 51 & 9.8 & 0.8 \\
\hline & Lithocarpus tubulosus (Hickel \& A.Camus) & Fagaceae & 79 & 15.2 & 0.8 \\
\hline & Lithocarpus dealbatus (Hook.f. \& Thomson ex Miq.) Rehder & Fagaceae & 51 & 9.8 & 0.6 \\
\hline & Gironniera subaequalis Planch & Cannabaceae & 79 & 15.2 & 0.6 \\
\hline & Syzygium zeylanicum (L.) DC. & Myrtaceae & 72 & 13.8 & 0.6 \\
\hline & Syzygium lanceolatum (Lam.) Wight \& Arn & Myrtaceae & 89 & 17.1 & 0.6 \\
\hline & Aphananthe cuspidata (Blume) Planch & Cannabaceae & 49 & 9.4 & 0.5 \\
\hline & Aphanamixis grandiflora & Meliaceae & 64 & 12.3 & 0.4 \\
\hline & Scaphium macropodum (Miq) Beumee ex K.Heyne & Malvaceae & 62 & 11.9 & 0.4 \\
\hline & Total (13) & & 823 & 158.3 & 10.3 \\
\hline & Other (104) & & 1543 & 296.7 & 15.2 \\
\hline
\end{tabular}

(Att = attribute; $\mathrm{N}=$ number of stem of a species in the attribute; $\mathrm{Abu}=$ Abundance; $\mathrm{Dom}=$ Dominance)

(Lam.) Wight \& Arn, Lithocarpus dealbatus (Hook.f. \& Thomson ex Miq.) Rehder, Alangium ridleyi King, Gironniera subaequalis Planch, Lithocarpus ducampii (Hickel \& A.Camus), Syzygium zeylanicum (L.) DC, Scaphium macropodum (Miq) Beumee ex K.Heyne, Aphanamixis grandiflora, Bursera tonkinensis Guillaum and Symplocos glauca (Thunb.) Koidz. But the most dominant species were $L$. dealbatus, B. tonkinensis, $S$. lanceolatum and Shorea roxburghii G.Don (Table 3).

At the steeper slope (S2), the ten most dominant species, out of the 117 present, were: Lithocarpus ducampii (Hickel \& A.Camus), Syzygium lanceolatum (Lam.) Wight \& Arn, Gironniera subaequalis Planch, Lithocarpus tubulosus (Hickel \& A.Camus), Syzygium zeylanicum (L.) DC, Aphanamixis grandiflora, Scaphium macropodum (Miq) Beumee ex K.Heyne, Engelhardtia roxburghiana Lindl, Lithocarpus dealbatus (Hook.f. \& Thomson ex Miq.) and Aphananthe cuspidata (Blume) Planch but the most dominant species were L. ducampii, Bursera tonkinensis Guillaum, Dipterocarpus grandiflorus Blanco and Shorea roxburghii G.Don (Table 3).

\subsection{Species diversity and Species distribution}

Table 1 shows significant differences $(\mathrm{p}<0.05)$ for mean number of tree species, number of families, tree density, and basal area (BA) per hectare between $\mathrm{H} 1$ and $\mathrm{H} 2$. The sample results show that the higher elevation zone had lower species diversity, families, and stem density; but higher BA. Meanwhile, there are no significant differences $(\mathrm{p}<0.05)$ in mean family richness and Evenness $(\mathrm{Eq})$ between S1 and S2. The mean values of number of species, family plot $^{-1}$, and tree density per hectare at the lower elevation and slope were higher than those of the higher elevation and slope except for number of families in $\mathrm{S} 1$ and $\mathrm{S} 2$.

The diversity of tree species, family, Shannon Index (H) and Evenness (Eq) of each topographic attribute presented in Table 1 indicating the species diversity in Shannon Index and its Evenness amongst attributes were different and showed the same trend of Shannon Index between elevation and slope. Even though, the mean Evenness between S1 and S2 was not significantly different at $(\mathrm{p}<0.05)$.

The results presented in Figure 2 show negative correlation of the number of species with elevation at -0.87 , with stem density at -0.60 , and with number of families at -0.77 . A weak positive correlation of 0.15 exists between BA and elevation. The results show weak negative correlation of slope with species, stem density and family at $-0.32,-0.19$ and -0.27 , respectively. Slope exhibits a very week positive correlation with BA (0.06). 


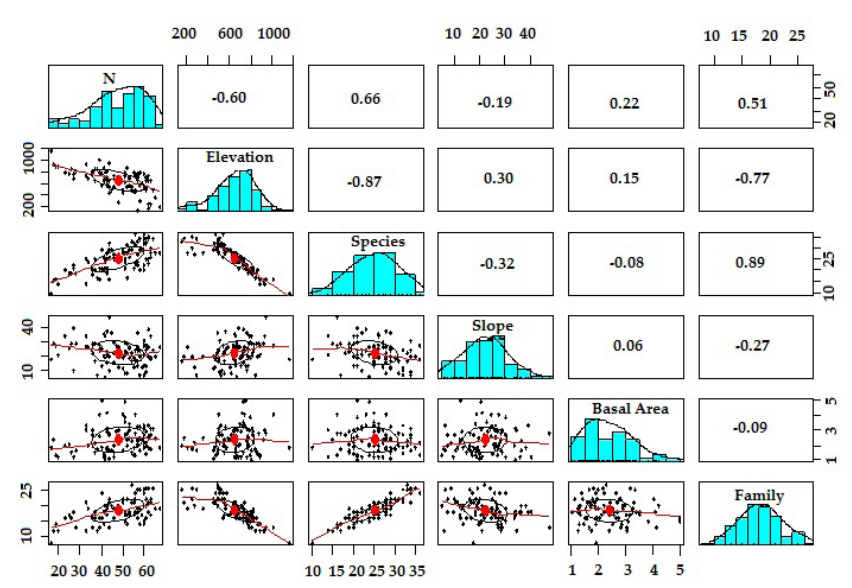

Figure 2. Paired correlation between elevation, slope with species, stem density $(\mathrm{N})$, basal area, family.

\subsection{Species composition}

The most ten dominant species of each topographic attribute were ranked (Table 2) according to species abundance as number of individuals (N/ha), absolute dominance as basal area $\left(\mathrm{m}^{2} / \mathrm{ha}\right)$, frequency in percent (\%), and IVI of each species. The most dominant species at different elevations are mapped in Figure 3 in which the ten most dominant species of the representative in $\mathrm{H} 1$ and $\mathrm{H} 2$ were presented based on the elevation levels ( $\mathrm{m}$ a.s.l.). The species composition provides information on the vegetation formation and contribution of each species through its abundance, dominance, and frequency, which help to explain the ecological habitat status in the study area (Table 2). The IVI is the combination of relative density, relative dominance and relative frequency in percentage, and helps rank the importance of a species in each attribute.

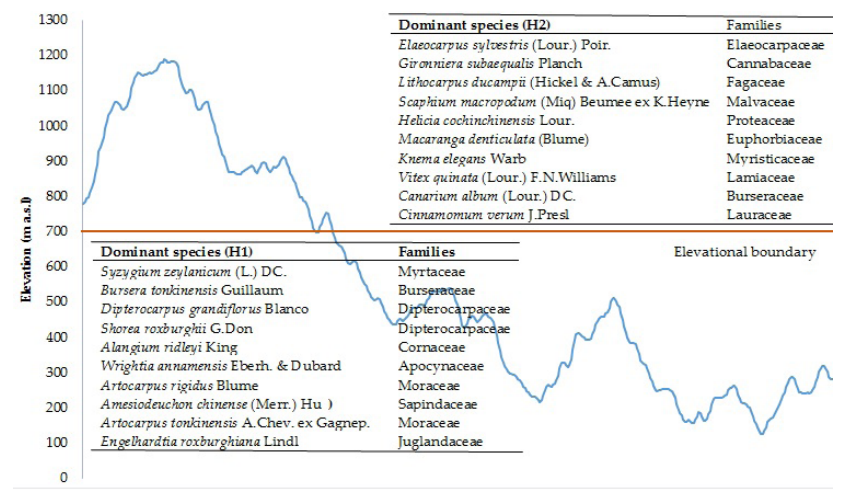

Figure 3. Most dominant species accounted in the representative plots of elevation levels $(\mathrm{H} 1$ and $\mathrm{H} 2)$.

\section{Discussion}

\subsection{Topographic Vegetation Distribution}

Obtaining knowledge on species richness, species diversity and the species distribution among different elevations and slopes is a powerful tool in plant ecology to determine species composition ${ }^{38}$. The most abundant and dominant species in $\mathrm{H} 1, \mathrm{H} 2, \mathrm{~S} 1$ and $\mathrm{S} 2$ are presented in Table 3 . The main species present in each attribute were determined, in primarily evergreen and semi- evergreen forest ${ }^{21}$. The number of species and number of families found in the current study, presented in Table 3, further emphasize the results of $\frac{38}{3}$ and $\stackrel{39}{~}$. The above listed dominant families and species found in the current study were naturally distributed in central Truong Son Mountain range, and were reported by many researchers $\frac{25,39,40}{}$. At the lower elevation (H1), the most abundant species presented in Table 3 were S. lanceolatum, L. ducampii, L. dealbatus, G. subaequalis, S. zeylanicum, A. ridleyi, S. macropodum, B. tonkinensis, L. tubulosus and S. glauca. Of those species, L. dealbatus, B. tonkinensis and L. ducampii were the most dominant.

In the higher elevation (H2), the most abundant species present were L. ducampii, S. roxburghii, D. grandifloras, B. tonkinensis, E. roxburghiana, L. tubulosus, G. subaequalis, S. zeylanicum, L. dealbatus, A. grandiflora, A. ridleyi, S. macropodum. Of these species, L. ducampii, S. roxburghii, D. grandifloras and S. zeylanicum were the most dominant and especially in the higher elevation, there were more endemic species from genera of Dipterocarpus, Shorea, Engelhardtia and Scaphium which are important and endemic of the northern Truong Son Mountain Range $e^{11,25}$.

The abundant and dominant species in both shallower slope (S1) and steeper slope (S2) were not much different among themselves and with $\mathrm{H} 1$ and $\mathrm{H} 2$ presenting in Table 3. But it is good to note that the steeper slope had more endemic species than those in shallower slope (i.e, three endemic species were found in S1 as Shorea, Engelhardtia and Scaphium but four endemic species found in S2 were B. tonkinensis, Dipterocarpus, Shorea, Engelhardtia and Scaphium. These patterns help explain that forests in both lower elevation and shallower slope could have more influences by humans than those in the higher elevation and steeper slope ${ }^{41,42}$. The endemic species were found more dominant and abundant in the higher elevation and steeper slope where has low disturbance and better management and protection ${ }^{43-45}$. 
The aim of establishment of protected in Vietnam is to protect, research on the main ecosystem, threatened and endemic species of flora and fauna including their

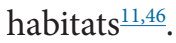

\subsection{Species diversity and Species distribution}

In the tropical area, the species richness, diversity and composition at the higher elevation were lower than at the lower elevation, meaning that the species richness, diversity decreased with an increase of elevation ${ }^{47}$. This relationship explains that forests at the lower elevation could be more environmentally shaped than those at the higher elevation. The lower elevation forests may be less productive due to competition or disturbance from humans and management ${ }^{48}$. The species richness between $\mathrm{H} 1$ and $\mathrm{H} 2$ was significantly different $(\mathrm{p}<0.05)$. Species richness declined with increasing elevation, which is in agreement with $\stackrel{31}{ }$ and $\stackrel{49}{9}$. The species richness was also significantly different $(\mathrm{p}<0.05)$ between $\mathrm{S} 1$ and $\mathrm{S} 2$, where S2 had lower species richness than S1. This indicates that the lower elevation and slope had more species richness, and that elevation had more influence than slope on species richness and diversity ${ }^{50,51}$. The species richness, diversity and composition in the study area could have been influenced significantly by the gradients $s^{52}$, not only with the number of species but also the number of families $(p<0.05)$. At the lower elevation and shallower slope, forests could be more fragmented since radiation can reach the forest floor, so more pioneer species can become dominant during succession ${ }^{53}$. The tree density between the different elevations and slopes was significantly different $(\mathrm{p}<0.05)$, this result corresponds to the findings of others $\frac{54-57}{}$. The BA was significantly different between $\mathrm{H} 1$ and $\mathrm{H} 2$, as well as between $\mathrm{S} 1$ and S2 $(p<0.05)$. Since the Basal Area (BA) is one of the elements contributing to species dominance in the forest stand and over topographic attributes. This finding is in line with the findings of some studies where the BA increased with an increase of elevation $\frac{59,60}{}$.

Shannon Index $(\mathrm{H})$ and Evenness $(\mathrm{Eq})$ ranged from 2.7 to 3.2 and from 93 to 98 in all topographic attributes, respectively. The species diversity and Evenness at the lower elevation and slope were higher than for the higher elevation and steeper slope $(\mathrm{p}<0.05)$, which corresponds to the species richness. The Shannon Index between $\mathrm{H} 1$ and $\mathrm{H} 2$ is similar to the findings of Sinha et al. (2018) significantly different (95\%). While the trend of Evenness
(Eq) at the steeper slope (S2) is higher than those in S1, it is not significantly different. The different consistence may show that the Evenness correlates to the disturbance, which is stronger in less steep topography ${ }^{48}$. Species diversity, basal area and species distribution presented in the current study can be differed from other studies such as ${ }^{50}$ within the tropical rain forest of Costa Rica, $\stackrel{31}{ }$ in the moist temperate forest of Garhwal Himalaya, and 51 in Sierra Nevada, Mexico due to the different temperature and rainfall and different range of elevations of tropical forests in the southeast Asia. Especially in Latin America where the deciduous forests accounted for $47 \%$ of forest $\operatorname{area}^{62}$.

\subsection{Species composition}

The most dominant species which contribute to the species composition of the representative plots for $\mathrm{H} 1$ and $\mathrm{H} 2$ was different. However, the S. zeylanicum species was the most abundant, numbering five individuals in the representative plot out of 63 individuals $(5: 63)$ in the entire topographic attribute of $\mathrm{H} 1$, the $B$. tonkinensis was not as dominant as $S$. zeylanicum in the presentative plot of $\mathrm{H} 1$ but it is more dominant than S. zeylanicum in the entire topographic attribute. As four out of the ten dominant species at the lower elevation $(\mathrm{H1})$ occupied $0.19 \mathrm{~m}^{2}$ out of $8.80 \mathrm{~m}^{2}$ per ha in representative plot, and $2.22 \mathrm{~m}^{2}$ out of $17.53 \mathrm{~m}^{2}$ per ha in the whole attribute, consisting of the Burseraceae, Diptercarpaceae and Juglandaceae families ${ }^{63}$. According to, the dominant species below $1000 \mathrm{~m}$ a.s.l. (in the south) and $700 \mathrm{~m}$ a.s.l. (in the north) are of Fagaceae, Myrtaceae, Lauraceae, Cannabaceae, Meliaceae, Malvaceae, Leguminosae; and Dipterocarpaceae, Burseraceae, Sapotaceae, Magnoliaceae, and Meliaceae; respectively. The dominance of these species confirms the stated forest type of moist and evergreen forests (where the annual rainfall is above $2500 \mathrm{~mm}$ and the moisture content is greater than $85 \%)^{64}$. Some of the predominant species, such as S. zeylanicum, A. ridleyi, W. annamensis, A. rigidus, $A$. chinense and $A$. tonkinensis, were dominant and light demanding at this elevation range, representing a type of secondary forest $t^{21}$. These ten species occupied $160 \%$ out of $300 \%$ of IVI in the representative plot and $42.27 \%$ out $300 \%$ of IVI of the entire topographic attribute. The comparison of the Important Value Index of the dominant species in the representative plots with those in the entire topography gave a modest explanation of species composition in the entire topographic attribute. The values for dominance and abundance of both the representative plots and the 
topographic attribute is an indicator of species diversity, as lower dominance and abundance values result in high diversity. At the higher elevation (H2), the dominant species were E. sylvestri, G. subaequalis and $L$. ducampii, which occupied $37.41 \%, 24.22 \%$ and $23.92 \%$ out of $175.72 \%$ in representative plot and occupied $50.5 \%$ out of $90.75 \%$ abundance and $3.22 \mathrm{~m}^{2}$ out of $4.87 \mathrm{~m}^{2}$ per ha $\mathrm{ha}^{-1}$ of the ten most dominant species in this range of elevation. In this plot, there were three S. macropodum individuals out of 52, this species is the multiple-use propose of this area $\mathrm{a}^{\frac{65}{}}$.

The composition of C. album, C. verum, L. ducampii, E. sylvestris, G. subaequalis indicates complex vegetation cover, where approximately 80 to 90 species can be commonly found in the fallow soil type or after being selectively-logged or fragmented. The dominant species in two representative plots of S1 and S2 could explain vegetation cover occurring in the lowland forests below $700 \mathrm{~m}$ (in the north) and 1000 $m$ a.s.l. (in the south), especially in the humid region of both the Northern and Southern Truong Son Mountain Range where a combination of Lauraceae, Fagaceae, Moraceae, Anacardiaceae families were commonly found ${ }^{25,39}$.

At steeper slope (S2), Lithocarpus ducampii are the most abundant and dominant, accounting for 32\% in the representative plot and $10.55 \%$ in the topographic attribute. In this ecological range, Engelhardtia roxburghiana accounted for $16.75 \%$, followed by Ormosia pinnata (15.66\%), Sapium discolor (14.39\%), and Gironniera subaequalis (10.98\%) in the representative plot. On the other hand, G. subaequalis had the second highest abundance in the whole attribute with 15.19 individuals per ha, followed by Aphanamixis grandiflora (12.31 individuals) and Engelhardtia roxburghiana (9.81 individuals).

\section{Conclusions}

The species richness, species diversity, stand density and basal area were significantly different between lower and higher elevations, as well as between shallower and steeper slopes. The lower elevation and shallower slope had more species richness and diversity than did the higher elevation and steeper slope. The species diversity Evenness (Eq) presented in both the lower elevation (H1) and slope (S1) was higher than those of higher elevation (H2) and slope (S2). The higher elevation and steeper slope had a larger mean basal area, but less individuals per ha, this could result from forest disturbance and successional processes. Elevation had more influence on species, stand density, species diversity, basal area and family distribution than those of the slope. Slope had a weak correlation with the species richness, stand density and basal area in the study area. Elevation was the key factor influencing species richness, stem density and species dominance in the study area.

Species composition between elevations and slopes was significantly different in term of species dominance and abundance. Difference of species composition could have been subjected to disturbance or successional processes of forest ecosystem in the current study area.

The species composition between slopes was not clearly distinguishable, this could help to confirm that slopes do not contribute to species distribution over topographic attributes.

The species composition of the most abundant and dominant species in the representative plots did not fully represent the species which were dominant and abundant at the entire topographic attribute in the study area. It could result from the low variation of topographic elevation or can be that the location of the representative plots favors for a certain microsite or aspects.

The current study can only report some major parameters of the forests; therefore, further efforts are needed for more information on endemic species and the most dominant species, which would contribute to appropriate conservation, restoration and management strategies in the area.

\section{Acknowledgements}

The authors would like to thank: the German Academic Exchange Service (DAAD); We acknowledge support of the German Research Foundation and the Open Access Publication Funds of the Göttingen University; the Department of Climate Change under the Ministry of Natural Resource and Environment of Vietnam; the University of Hue Agriculture and Forestry for their help in arranging administrative permission to access the research areas; the experts of Sub- Forest Inventory and Forest Planning in Thua Thien Hue province; We would like to express our sincere thanks to Prof. Dr. Ralph Mitlöhner for his valuable advice and to the forest experts of Forest Management Board of the A Luoi and Rangers of Sao La Nature Reserve for their during different phases of fieldwork; the botanists for tree species identification; and local authorities and local people in the A Luoi district. We thank Department of Cartography, GIS and Remote Sensing, Göttingen University for providing the ArcGIS software in the framework of research. We specially thank editors and reviewers for constructive comments for publication assistance. 


\section{References}

1. Ashton PS, Hall P. Comparisons of structure among mixed dipterocarp forests of North-Western Borneo. Journal of Ecology. 1992; 80(3):459-81. https://doi. org/10.2307/2260691

2. An introduction to tropical rain forests. [Internet]. [cited 1990]. Available from: https://global.oup.com/academic/ product/an-introduction-to-tropical-rain-forests$9780198501473 ? \mathrm{cc}=\mathrm{in} \& \mathrm{lang}=\mathrm{en} \&$.

3. McIntosh RP. An index of diversity and the relation of certain concepts to diversity. Journal of Ecology. 1967; 48(3):392-404. https://doi.org/10.2307/1932674

4. Hall JB, Swaine MD. Classification and Ecology of ClosedCanopy Forest in Ghana. Journal of Ecology. 1976, 64 (3):913-951. https://doi.org/10.2307/2258816

5. Huang W, Pohjonen V, Johansson S, Nashanda M, Katigula MIL, Luukkanen O. Species diversity, forest structure and species composition in Tanzanian tropical forests. Forest Ecology and Management. 2003; 173(1-3):11-24. https:// doi.org/10.1016/S0378-1127(01)00820-9

6. Anbarashan M, Parthasarathy N. Tree diversity of tropical dry evergreen forests dominated by single or mixed species on the Coromandel coast of India. Journal of Tropical Ecology. 2013; 54(2):179-90.

7. Brown RC, Frederichsen TS. Topographic factors affecting the tree species composition of forests in the upper piedmont of Virginia. Virginia Journal of Science. 2008; 59(1):1-9.

8. Bunyavejchewin S, LaFrankie JV, Baker PJ, Kanzaki M, Ashton PS, Yamakura T. Spatial distribution patterns of the dominant canopy dipterocarp species in a seasonal dry evergreen forest in western Thailand. Forest Ecology and Management. 2003; 175(1-3):87-101. https://doi. org/10.1016/S0378-1127(02)00126-3

9. Kacholi DS. Edge-interior disparities in tree species and structural composition of the kilengwe forest in Morogoro Region, Tanzania. International Scholarly Research Notices; 2014. p. 1-8. https://doi.org/10.1155/2014/873174

10. Thomas E, Vandebroek I, van Damme P. Valuation of forests and plant species in indigenous territory and National Park Isiboro-Sécure, Bolivia. Economic Botany. 2009; 63(3): 229-41. https://doi.org/10.1007/s12231-009-9084-5

11. Demography of threatened tree species in Vietnam [Internet]. [cited 2006]. Available from: file://C:/Users/a/ Downloads/full.pdf.
12. Forest rehabilitation in Vietnam: Histories, realities and future [Internet]. [cited 2006]. Available from: https://www. cifor.org/publications/pdf_files/Books/BDeJong0601.pdf.

13. Olson DM, Dinerstein E, Wikramanayake ED. Terrestrial ecoregions of the world: A new map of life on earth. BioScience. 2001; 51(11):933-8. https://doi. org/10.1641/0006-3568(2001)051 [0933:TEOTWA]2.0 .CO;2

14. Clark DA, Clark DB. Assessing the growth of tropical rain forest trees: Issues for forest modelling and management. Journal of Applied Ecology. 1999; 9(3):981-97. https:// doi.org/10.1890/1051-0761(1999)009[0981:ATGOTR]2.0 .CO;2

15. Trejo I, Dirzo R. Deforestation of seasonally dry tropical forest. Biological Conservation. 2000, 94(2):133-42. https:// doi.org/10.1016/S0006-3207(99)00188-3

16. María CRJ, Aide TM. Vegetation structure, species diversity, and ecosystem processes as measures of restoration success. Forest Ecology and Management. 2005; 218(1-3):159-73. https://doi.org/10.1016/j.foreco.2005.07.008

17. Chazdon RL, Letcher SG, van Breugel M, MartínezRamos M, Bongers F, Finegan B. Rates of change in tree communities of secondary Neotropical forests following major disturbances. Philosophical Transactions of the Royal Society B: Biological Sciences. 2007; 362(1478):273-89. https://doi.org/10.1098/rstb.2006.1990. PMid:17255036. PMCid:PMC2311434

18. Desta F, Colbert JJ, Rentch JS, Gottschalk KW. Aspect induced differences in vegetation, soil, and microclimatic characteristics of an Appalachian Watershed. Southern Appalachian Botanical Society. 2004; 69:92-108. https:// doi.org/10.2179/0008-7475(2004)069<0092:AIDIVS $>2.0$ .CO;2

19. Jucker T, Boris B, David FRP. Burslem. Topography shapes the structure, composition and function of tropical forest landscapes. Ecology Letter. 2018; 21:989-1000. https://doi. org/10.1111/ele.12964 PMid:29659115

20. Designing mixed species tree plantations for the tropics: Balancing Ecological Attributes of Species with Landholder Preferences in the Philippines [Internet]. [cited 2014 Apr 21]. Available from: https://journals.plos.org/plosone/ article?id=10.1371/journal.pone.0095267.

21. Nguyen HH, Diez JU, Kerstin W. Spatial distribution and association patterns in a tropical evergreen broad-leaved forest of north-central Vietnam. Journal of Vegetation Science. 2016; 27:318-27. https://doi.org/10.1111/jvs.12361 
22. Do HTT, Grant JC, Trinh BN, Zimmer HC, Nichols JD. Diversity depends on scale in the forests of the Central Highlands of Vietnam. Journal of Asia-Pacific Biodiversity. 2017; 10:472-88. https://doi.org/10.1016/j.japb.2017.08.008

23. The Country and Its Geographical Regions [Internet]. [cited 1997]. Available from: https://www.internetworldstats. com/list1.htm.

24. Nguyen TL, Florimond DS. Analysis and mapping of rainfall-induced landslide susceptibility in A Luoi District, Thua Thien Hue Province, Vietnam. Water. 2019; 11:51-66. https://doi.org/10.3390/w11010051

25. Le NTT. Adding some vascular plants to the list of plants in Thua Thien Hue Province. Journal of Research and Development. 2016; 4:96-107.

26. Pham TG, Nguyen HT, Kappas M. Assessment of soil quality indicators under different agricultural land uses and topographic aspects in Central Vietnam. International Soil and Water Conservation Research. 2018; 6:280-8. https:// doi.org/10.1016/j.iswcr.2018.08.001

27. Tran H, Shigeru IIDA, Inoue S. Species composition, diversity and structure of secondary tropical forests following selective logging in Huong Son, Ha Tinh Province, Vietnam. Journal of the Faculty of Agriculture. 2005; 50:551-71.

28. Forest Mensuration [Internet]. [cited 2007]. Available from: http://www2.ca.uky.edu/forestry/for250/Forest $\% 20$ Mensuration\%20book.pdf.

29. KalwijJM. Review of “The Plant List", a working list of all plant species. Journal of Vegetation Science. 2012; 23:998-1002. https://doi.org/10.1111/j.1654-1103.2012.01407.x

30. Mazier D, Baumgartner F, Lepitre C. Terrain classification for tropical logging: Application in the case of Gabon. Bois Forests des Tropiques. 1975; 162:47-67.

31. Sharma CM, Suyal S, Gairola S, Ghildiyal SK. Species richness and diversity along an altitudinal gradient in moisttemperate forest of Garhwal Himalaya. American Journal of Science. 2009; 5:119-28.

32. Adekunle VAJ, Olagoke AO, Akinele SO. Tree species diversity and structure of a Nigerian strict nature reserve. Journal of Tropical Ecology. 2013; 54:275-89.

33. The Mathematical Theory of Communication [Internet]. [cited 1964]. Available from: http://math.harvard. edu/ ctm/home/text/others/shannon/entropy/entropy. pdf.

34. Pielou EC. The measurement of diversity in different types of biological collections. Journal of Theoretical Biology. 1966; 13:131-44. https://doi.org/10.1016/00225193(66)90013-0

35. Procedures for psychological, psychometric, and personality research [Internet]. [cited 2013 Jan]. Available from: https://www.researchgate.net/publication/281345624_
psych_Procedures_for_Psychological_Psychometric_and_ Personality_Research_R_Package_Version_10-95.

36. Meireles LD, Shepherd GJ. Structure and floristic similarities of upper montane forests in Serra Fina mountain range, southeastern Brazil. Acta Botanica Brasilica. 2015; 29: 58-72. https://doi.org/10.1590/0102-33062014abb3509

37. Curtis JT, McIntosh RP. The interrelations of certain analytic and synthetic phytosociological characters. Ecology. 1950; 31:434-55. https://doi.org/10.2307/1931497

38. Naidu MT, Kumar OA. Tree diversity, stand structure, and community composition of tropical forests in Eastern Ghats of Andhra Pradesh, India. Journal of Asia-Pacific Biodiversity. 2016; 9:328-34. https://doi.org/10.1016/j. japb.2016.03.019

39. Van YT, Cochard R. Tree species diversity and utilities in a contracting lowland hillside rainforest fragment in Central Vietnam. Forest Ecosystems. 2017; 4:115-20. https://doi. org/10.1186/s40663-017-0095-x

40. Soejima A, Tagane S, Nguyen Van N, Duy CN, Huong NTT, Yahara T. Callicarpa bachmaensis Soejima and Tagane (Lamiaceae), a new species from Bach Ma National Park in Thua Thien Hue Province, Central Vietnam. PhytoKeys. 2016; 62:33-9. https://doi.org/10.3897/phytokeys.62.7974. PMid:27212880. PMCid:PMC4856901.

41. Hadley KS. The role of disturbance, topography, and forest structure in the development of a montane forest landscape. Bulletin of the Torrey Botanical Club. 1994; 121:47-61. https://doi.org/10.2307/2996883

42. Sébastien L, John, D. Thompson, Eric G, Max D. The biology and ecology of narrow endemic and widespread plants: A comparative study of trait variation in 20 congeneric pairs. OIKOS. 2004; 107:505-18. https://doi.org/10.1111/j.00301299.2004.13423.x

43. Saving nature's legacy: protecting and restoring biodiversity [Internet]. [cited 1994 Apr 01]. Available from: https:// www.amazon.com/Saving-Natures-Legacy-ProtectingBiodiversity/dp/1559632488.

44. Yocom LL, Fulé PZ. Human and climate influences on frequent fire in a high-elevation tropical forest. Journal of Applied Ecology. 2012; 49:1356-64. https://doi. org/10.1111/j.1365-2664.2012.02216.x

45. Fabrucius C, Burger M, Hockey PAR. Comparing biodiversity between protected areas and adjacent rangeland in xeric succulent thicket, South Africa: Arthropods and reptiles. Journal of Applied Ecology. 2003; 40:392-403. https://doi.org/10.1046/j.1365-2664.2003.00793.x

46. An LT, Markowski J, Bartos M. The comparative analyses of selected aspects of conservation and management of Vietnam's national parks. Journal for Nature Conservation. 2018; 25:1-30. https://doi.org/10.3897/ natureconservation.25.19973 
47. Sabatini FM, Borja JA, Sabina B, Andrea L, Milan C. Betadiversity of central European forests decreases along an elevational gradient due to the variation in local community assembly processes. Ecography. 2018; 41:1038-48. https:// doi.org/10.1111/ecog.02809

48. Zhang JT, Xu B, Li M. Vegetation patterns and species diversity along elevational and disturbance gradients in the Baihua Mountain Reserve, Beijing, China. Mountain Research and Development. 2013; 33:1-15. https://doi. org/10.1659/MRD-JOURNAL-D-11-00042.1

49. Gairola S, Sharma CM, Ghildiyal SK, Suyal S. Tree species composition and diversity along an altitudinal gradient in moist tropical montane valley slopes of the Garhwal Himalaya, India. Forest Science and Technology. 2011; 7:91-102. https://doi.org/10.1080/21580103.2011.597109

50. Tropical rain forest structure, tree growth and dynamics along a 2700-m Elevational transect in Costa Rica [Internet]. [cited 2015 Apr 09]. Available from: https://journals.plos. org/plosone/article?id=10.1371/journal.pone.

51. Sánchez AG, López LM. Plant species richness and diversity along an altitudinal gradient in the Sierra Nevada, Mexico. Diversity and Distributions. 2005; 11:567-75. https://doi. org/10.1111/j.1366-9516.2005.00186.x

52. Dorji T, Stein RM, Julia AK, Totland O. Plant species richness, evenness, and composition along environmental gradients in an alpine meadow grazing ecosystem in Central Tibet, China. Arctic Antarctic and Alpine Research. 2014; 46:308-26. https://doi.org/10.1657/1938-4246-46.2.308

53. Emery S. Succession: A closer look. Nature Education Knowledge Project. 2010; 3:45-50.

54. Factors determining forest diversity and biomass on a tropical volcano, Mt. Rinjani, Lombok, Indonesia [Internet]. [cited 2013 Jul 23]. Available from: https://journals.plos. org/plosone/article?id=10.1371/journal.pone.

55. Ifo SA, Moutsambote JM, Koubouana F. Tree species diversity, richness, and similarity in intact and degraded forest in the tropical rainforest of the Congo Basin: Case of the Forest of Likouala in the Republic of Congo. International Journal of Forestry Research; 2016. p. 1-12. https://doi.org/10.1155/2016/7593681

56. Sahu SC, Dhal NK, Rama RC. Tree species diversity, distribution and population structure in a tropical dry deciduous forest of Malyagiri hill ranges, Eastern Ghats, India. Tropical Ecology. 2012, 53, pp. 163-168.
57. Campbell DG, Stone JL, Rosas AJR. A comparison of the phytosociology and dynamics of three floodplain (Várzea) forests of known ages, Rio Juruá, western Brazilian Amazon. Botanical Journal of the Linnean Society. 1992; 108:213-37. https://doi.org/10.1111/j.1095-8339.1992.tb00240.x

58. Whitmore TC, Sidiyasa K. Composition and structure of a lowland rain forest at Toraut, Northern Sulawesi. Kew Bulletin. 1986; 41:747-57. https://doi.org/10.2307/4103127

59. Toledo GM, Williams LG. Tree diversity patterns in successive vegetation types along an elevation gradient in the Mountains of Eastern Mexico. Ecological Research. 2014; 29:1097-104. https://doi.org/10.1007/s11284-0141196-4

60. Chris C. The environmental control of plant species density on Himalayan elevation gradient. Journal of Biogeography. 2005; 32:999-1018. https://doi.org/10.1111/j.13652699.2005.01249.x

61. Sinha S, Badola HK, Bijoy C, Gaira KS, Lepcha J, Pitamber PD. Effect of altitude and climate in shaping the forest compositions of Singalila National Park in Khangchendzonga Landscape, Eastern Himalaya, India. Journal of Asia-Pacific Biodiversity. 2018; 11:267-75. https://doi.org/10.1016/j.japb.2018.01.012

62. Toledo TA, García FO. Effects of forest-pasture edge on $\mathrm{C}, \mathrm{N}$ and $\mathrm{P}$ associated with Caesalpinia eriostachys, a dominant tree species in a tropical deciduous forest in Mexico. Ecological Research. 2008; 23:271-80. https://doi. org/10.1007/s11284-007-0373-0

63. Lin L, Huang Z, Ye W. Spatial distributions of tree species in a subtropical forest of China. OIKOS. 2009; 118:495-502. https://doi.org/10.1111/j.1600-0706.2009.16753.x

64. Some considerations on the edaphic conditions in the tropical forest areas and their implications to land management [Internet]. [cited 1977]. Available from: http://horizon.documentation.ird.fr/exl-doc/pleins_textes/ pleins_textes_5/b_fdi_00/00065.pdf.

65. Growth, demography and stand structure of Scaphium macropodum in differently managed forests in Vietnam [Internet]. [cited 2012]. Available from: https://www. tropenbos.org/resources/publications/growth\%2C\%2Bde mography\%2Band\%2Bstand\%2Bstructure $\% 2$ Bof $\% 2$ Bscap

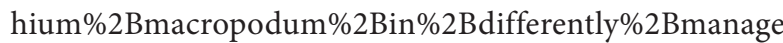
d\%2Bforests\%2Bin\%2Bvietnam. 